\title{
Lyapunov based Model Reference Adaptive Control for Aerial Manipulation
}

\author{
Matko Orsag, Christopher Korpela, Stjepan Bogdan, and Paul Oh
}

\begin{abstract}
This paper presents a control scheme to achieve dynamic stability in an aerial vehicle with dual multi-degree of freedom manipulators using a lyapunov based model reference adaptive control. Our test flight results indicate that we can accurately model and control our aerial vehicle when both moving the manipulators and interacting with target objects. Using the Lyapunov stability theory, the controller is proven to be stable. The simulation results showed how the MRAC is capable of stabilizing the oscillations produced from the unstable PI-D attitude control loop. Finally a high level control system based on a switching automaton is proposed in order to ensure the saftey of the aerial manipulation missions.
\end{abstract}

\section{INTRODUCTION}

Historically, UAV research has been focused on avoiding interaction with the environment. Recently, the focus has shifted towards aerial manipulation and bringing the unmanned aerial systems in contact with their surroundings. The ability to manipulate objects will, ultimately, greatly expand the use of unmanned aerial systems. Although some research groups tested various forms of adaptive control on quadrotor UAVs [1], [2], its full potential can be exposed through aerial manipulation missions, where the moment of inertia and the center of mass constantly fluctuate. Model reference adaptive control concept [3], proposed in this paper has been used in numerous applications [4]-[6]. Together with the original PID controller of the aircraft it is used to assure aircraft stability throughout the manipulation process.

Current State of the art in Aerial manipulation has focused mostly on single DOF gripper manipulators and slung load transport. A lot of researchers that contributed to this field of aerial robotics have so far focused on 3 key aspects:

- Contact inspection (Pose/Wrench control)

- Slung load transport

- Single Degree of freedom grippers

This material is based on research sponsored in part by the Air Force Research Laboratory, under agreement number FA8655-13-1-3055 and European Community Seventh Framework Program under grant No. 285939 (ACROSS). The U.S. Government is authorized to reproduce and distribute reprints for Governmental purposes notwithstanding any copyright notation thereon.

M. Orsag and Stjepan Bogdan are with Faculty of Electrical Engineering and Computing, University of Zagreb, 10000 Zagreb, Croatia \{morsag, sbogdan\}efer.hr

C. Korpela and P. Oh are with the Drexel Autonomous Systems Lab, Drexel Univeristy, Philadelphia, PA 19104 USA \{cmk325, yj55, pkb471\}@drexel.edu, paul@coe.drexel.edu

The views and conclusions contained herein are those of the authors and should not be interpreted as necessarily representing the official policies or endorsements, either expressed or implied, of the Air Force Research Laboratory or the U.S. Government.

\section{A. Pose/Wrench control}

In [7], authors introduced wrench control to give the quadrotor an ability of stable motion while in contact. The authors used a hybrid Pose/Wrench framework capable of switching between pure Pose and Pose/Wrench control but the operator is required to make the switch. Instead of using additional force/torque sensors, authors utilized a wrench estimator using quadrotor inputs and pose measurements. Another hybrid Force/Position control concept based on state feedback is introduced in [8], [9]. In this work, a duct-fan aerial vehicle is used to achieve contact inspection tasks. The authors presented a mechanical design to cope with inherent zero dynamics of the system.

\section{B. Slung Load}

Authors in [10] explored the possibility of using single and multiple UAVs to assist in search and rescue (SAR) missions. They tested formations of up to three small size petrol powered helicopters that cooperatively transport a slung load. The authors were capable of transporting a video camera with three small size helicopters in adverse weather conditions that exhibited high wind speeds of up to $35 \mathrm{~km} / \mathrm{h}$. A group of authors in [11] implemented an additional vision system that measured the position of the slung load. They proposed an adaptive controller that reduced the swing in the load. In order to solve a similar problem with quadrotors carrying a slung load, authors in [12] proposed a technique based on dynamic programming which ensured swing free trajectory tracking.

In [13], a tethered helicopter configuration is modeled and tested. This configuration proved to be more stable in

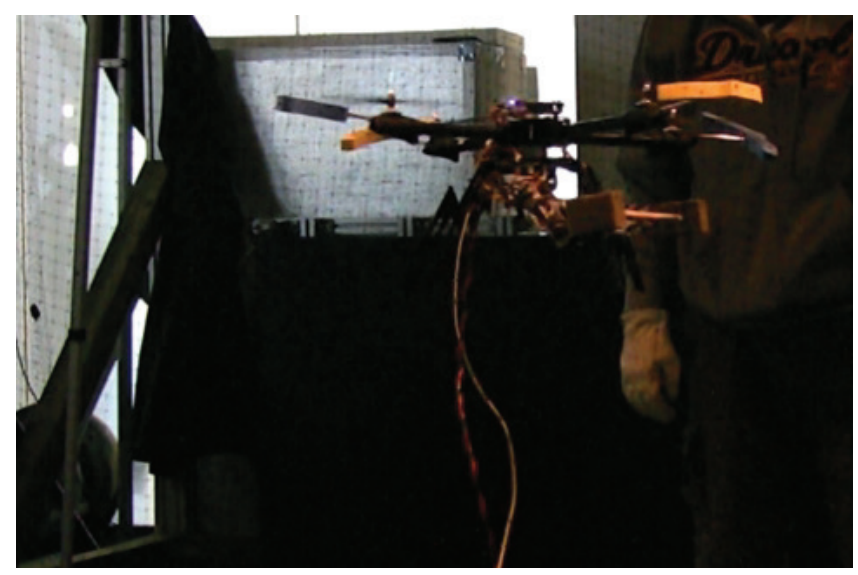

Fig. 1: MM-UAV carrying a long rod 
presence of disturbances (i.e. wind gusts) then a non-tethered helicopter. Tether also introduced coupled dynamics, adding to the complexity of the overall controller. Nevertheless, tether is a very interesting concept that could potentially be used in twofold manner: It could provide unlimited supply of electricity; It could also be used as additional pose and position measurement system.

\section{Single DOF Grippers}

The authors in [14]-[16] analyzed the stability of a helicopter and a quadrotor with added payload mass. Using Routh-Hourwitz criteria, the authors derived a connection between the mass off the added payload, its offset from UAV center of mass and the stability of the UAV. Plotting offset vs. mass stability regions, they clearly showed how a bigger mass tightens the stability region of the aircraft. Namely, the bigger the mass, the smaller the available offset region, so that the vehicle has to grab the additional payload as close to the center of gravity as possible. Quadrotor stability in presence of unknown payload disturbances was discussed in [17]. Here the authors look into the possibility of estimating disturbance parameters (i.e. mass and moments of inertia). Using hover mode to estimate the parameters, they effectively eliminated the Euler angles and the derivatives of the position from the equations. This research group also contributed by doing an experimental study with teams of quadrotors cooperatively grasping, stabilizing, and transporting payloads along desired three-dimensional trajectories [18]. They went a step further and showed the experimental results of team of quadrotors performing automated assembly of Special Cubic type Structures [19]. They used their gripping tool to pick up the simple structural nodes and used magnetic endings on the structures to piece them together.

\section{Beyond the current State of the Art}

The aim of this research is to extend the current state of the art by introducing multiple degree of freedom manipulators. To the extent of authors knowledge, little or no attempt has been made to implement such an aerial vehicle so far. Afore mentioned state of the art is limited to simple manipulation problems (i.e. Pick and place, contact inspection, painting, etc.). Multiple - Degree of Freedom manipulators expand the capabilities of aerial robots by giving them the ability to: Perch and manipulate, Twist valves on or off, Assemble objects, remove obstacles and many other.

Introducing multiple degrees of freedom adds to the complexity of the control problem. Therefore, the first goal of this research is to achieve and sustain a stable flight while moving manipulator arms. A lot of research in mobile robotics as well as the research in air robots with 1DOF grippers can be utilized to solve this problem. After this, next research step would be to implement current state of the art in contact stability and load estimation which would yield a fully dexterous aerial robot. The following two chapters present a first step towards a fully dexterous MM-UAV.

\begin{tabular}{ccccc}
\hline Link & $\theta$ & $d$ & $a$ & $\alpha$ \\
\hline $\mathrm{B}-0$ & $\frac{\pi}{2}$ & $d_{b}$ & $a_{b}$ & 0 \\
0 & 0 & 0 & 0 & 0 \\
1 & $q_{A}^{1}-\frac{\pi}{2}$ & 0 & 3.75 & $-\frac{\pi}{2}$ \\
2 & $q_{A}^{2}$ & 0 & 3.75 & $\frac{\pi}{2}$ \\
3 & $q_{A}^{3}$ & 0 & 3.75 & $-\frac{\pi}{2}$ \\
4 & $q_{A}^{4}+\frac{\pi}{2}$ & 0 & 0 & $\frac{\pi}{2}$ \\
T-E & 0 & 0 & 3.75 & 0 \\
\hline
\end{tabular}

TABLE I: Denavit-Hartenberg Parameters for Manipulators $[\mathrm{cm}]$. Showing Arm A only for clarity.

\section{Manipulator Model}

Using the recursive Newton-Euler approach and DenavitHartenberg parametrization for forward kinematics, each arm is modeled as a serial chain RRRR manipulator [20]. The coonection between the quadrotor body frame and the first joint of each arm is represented with static revolute joint with a constant angular offset for each MM-UAV arm (Link $B-0)$. Apllying the results from [21] quadrotor dynamics are introduced to the aerial platform of the robot.

Denavit-Hartenberg (DH) parameters of the manipulator arms are shown in Table I. Parameters $\theta, d, a$, and $\alpha$ are in standard DH convention and $q_{i}^{1}, q_{i}^{2}, q_{i}^{3}$, and $q_{i}^{4}$ are joint variables of each manipulator arm $i=[A, B]$. Since the whole aircraft is symmetrical, the general kinematic structure is identical for the right and left arms, the coordinate frames are the same for each arm, and only the link $B-0$ is different for the two arms. Reference frames are shown in Fig. 2 which relate the world frame, $W$, via body frame, $B$, to the endeffector frame, $E$. To make the $\mathrm{DH}$ parameters consistent, an additional, virtual frame $T$ is set in the origin of frame $L_{4}$. The direct kinematics function relating the quadrotor body to the end-effector frame is obtained by chain-multiplying the transformation matrices together:

$$
\mathbf{T}_{b}^{e}(q)=\mathbf{T}_{b}^{0} \mathbf{T}_{0}^{4} \mathbf{T}_{4}^{e}
$$

With Denavit-Hartenberg parameterization, joint frames are set and direct kinematics equations for each serial chain are derived. This procedure is repeated for both manipulator arms. Given the initial angular $\Omega_{B}$ and translational $V_{B}$ velocities of the quadrotor body, the angular $\vec{\omega}_{j}^{i}$ and translational $\vec{v}_{j}^{i}$ velocities and the derivatives of the velocity vectors (i.e. $\dot{v}_{j}^{i}$ and $\dot{\omega}_{j}^{i}$ ) for each joint $j$ and each arm $i$, can be propagated and expressed in the quadrotor body frame.

\section{QUADROTOR MODEL}

This paper tries to combine both manipulator dynamics and the dynamics of the quadrotor. Due to the resulting complexity of model and mission requirenments, the quadrotor dynamics considered in this paper do not account for various aerodynamic effects (i.e. blade flapping, ground effect, etc.) experienced during highly dynamic flying maneuvers. Most of the missions require stable hovering maneuvers, which justifies a simplified mathematical model without accounting for the previously mentioned aerodynamic effects.

As the manipulator dynamics are introduced through the recursive Newton-Euler method, it is possible to separate 


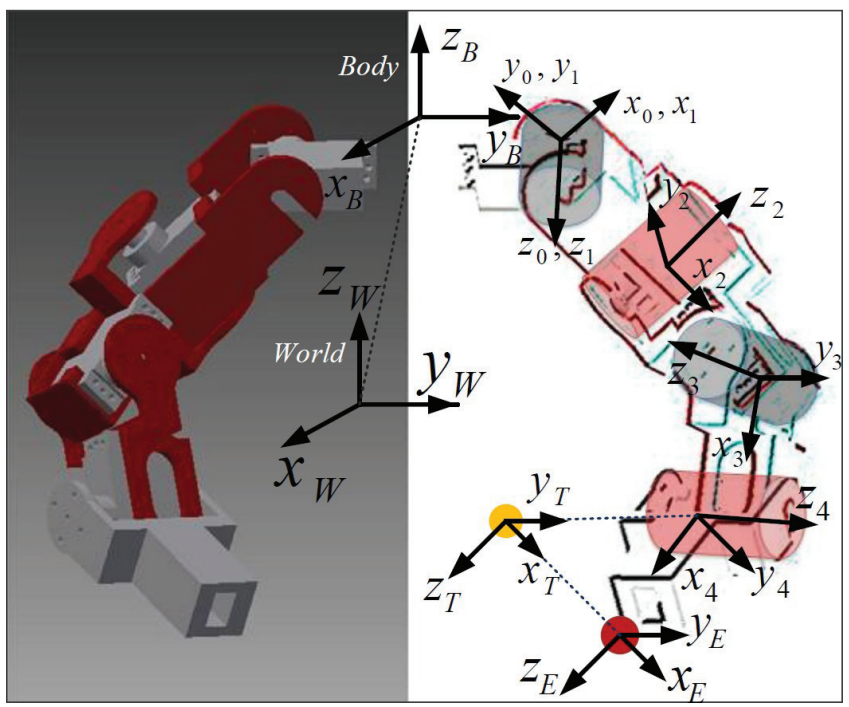

Fig. 2: Reference Frames for Manipulator Arms

model the quadrotor motion based on Newton-Euler equations for rigid body translation and rotation [22]. The mass, moments of inertia, and dynamic movement of the manipulator are then introduced as disturbances to the quadrotor model.

$$
\left[\begin{array}{c}
\vec{F}_{q} \\
\vec{\tau}_{q}
\end{array}\right]=\left[\begin{array}{cc}
m_{q} \mathbf{I} & 0 \\
0 & \mathbf{J}_{q}
\end{array}\right]\left[\begin{array}{l}
\overrightarrow{\dot{v}} \\
\overrightarrow{\dot{\omega}}
\end{array}\right]+\left[\begin{array}{c}
0 \\
\vec{\omega}_{q} \times \mathbf{J}_{q} \vec{\omega}_{q}
\end{array}\right]
$$

In the first aerodynamic approximation, rotor thrust and torques $\vec{T}$ and $\vec{Q}$, respectively are proportional to the square of rotor speed $\Omega$, which is consequently proportional to the voltage $\Omega \propto U[V]$. Forces and torques of each propeller are added according to the standard quadrotor propulsion system equations as shown in (3).

$$
\begin{gathered}
\overrightarrow{F_{t o t}}=\overrightarrow{T^{1}}+\overrightarrow{T^{2}}+\overrightarrow{T^{3}}+\overrightarrow{T^{4}} \\
\tau_{x}^{\text {tot }}=\tau_{x}^{2}+\tau_{x}^{3}-\tau_{x}^{1}-\tau_{x}^{4} \\
\tau_{y}^{t o t}=\tau_{y}^{3}+\tau_{y}^{4}-\tau_{y}^{1}-\tau_{y}^{2} \\
\tau_{z}^{\text {tot }}=\tau_{z}^{2}+\tau_{z}^{4}-\tau_{z}^{1}-\tau_{z}^{3}
\end{gathered}
$$

A lot of researchers usually neglect the underlying dynamics of brushless DC motors used on the available quadrotor platforms. For some missions and for a very few available platforms, this symplifycation proved to be acceptable. However, in the presence of the added payload and its inertia, as well as the mission requirenments for air robots, the dynamics of the motors has an important impact in the overall aircraft stability and cannot be omitted from the MM-UAV model. Off the shelf electronic speed controllers are used to power and control the motors, which makes it impossible to devise a complete model for the motors. Therefore, a simplified 1st order PT1 dynamic model is used.

Considering simplified aerodynamic conditions, propellers simply produce thrust forces $\vec{T}^{i}$. Summing them all together gives the total aircraft thrust. Each propeller torque $\vec{\tau}^{i}$, in contrast, has two components, one coming from the actual propeller drag, and the other due to the displacement of the propeller from the center of mass.

$$
\vec{\tau}^{i}=\vec{Q}^{i}+\Delta \vec{R}_{T}^{i} \times \vec{T}^{i}
$$

\section{STABILITY BOUNDS}

Previous work in this field has concentrated only on load mass stability problem, ignoring the coupled manipulator dynamics [17], [15]. For a 1DOF gripper tools, this assumtion is welcome, but for 4-DOF arms that introduce a significant increase in payload and moment of inertia tihs symplification cannot be applied. Therefore, two underlying effects are identified to cause the change in the overall center of mass $\overrightarrow{C M}$ and the overall moment of inertia $\mathbf{I}_{C M}$ :

- Repositioning of the arms

- Load mass and moment of inertia

Building upon the results from [23], [24] we present a simplified arm model to establish a stability criteria for an ilustrative air robot mission example: Picking up and manipulating a long cylinder type object Fig $3 a$ and $3 b$.

\section{A. Center of Mass and Moment of Inertia distribution}

In this simplified kinematics model (Figs. 3a and 3b), only the movement of the second joint in both arms are considered (i.e. $q_{A}^{2}$ and $q_{B}^{2}$ ). The rest of the arm joints remain fixed. With this simplification it is possible to view the arms as links of length $2 C$ and mass $m_{A}, m_{B}$ respectively, and the corresponding moments of inertia.

As shown in [24], one can easily calculate the varying center of mass $C M$ :

$C \vec{M}=\frac{\vec{Q}_{c m} m_{Q}+\vec{A}_{c m}(t) m_{A}+\vec{B}_{c m}(t) m_{A}+\vec{L}_{c m}(t) m_{L}(t)}{m_{Q}+m_{A}+m_{B}+m_{L}(t)}$

The overall moment of inertia $C M$ changes as the joints move. This variation can easily be derived using the Parallel axis theorem:

$$
\begin{aligned}
& I_{c m}=I_{Q}+R_{0}^{e}(t)\left(I_{A}+I_{B}+I_{L}\right) R_{0}^{e}(t)^{T} \\
& +m_{Q} \Delta Q(t)^{2}+m_{A} \Delta A(t)^{2}+m_{B} \Delta B(t)^{2}+m_{L}(t) \Delta L(t)^{2}
\end{aligned}
$$

Where $\Delta Q, \Delta A \Delta B$ and $\Delta L$ represent the center of mass of each body with respect to the overall center of mass $C M$. These vectors are a function of time because they change as the joints $q_{A}^{2}$ and $q_{B}^{2}$ change through time. $\mathbf{I}_{Q}$ is the quadrotor body moment of inertia, written in the base coordinate system, and $\mathbf{I}_{A}, \mathbf{I}_{B}$ and $\mathbf{I}_{L}$ represent the moments of inertia for each arm and the load written in the endeffector's frame of reference. The transformation matrix $\mathbf{R}_{0}^{e}(t)$, extracted from (1), transforms these moments of inertia into the quadrotor base coordinate system.

Final equations for the center of mass $C M$ and the moment of inertia around it $J_{C M}$ are too complex to be presented in analytic form, therefore the overall changes in $\mathbf{I}_{C M}$ are shown in Fig 4. The images are plotted relative to the quadrotor moment of inertia $\mathbf{I}_{Q}$. Although the effects of each joint change are similar, there are a few substantial differences: Joints 1 cause a greater shift in $C \vec{M}$ then Joints 


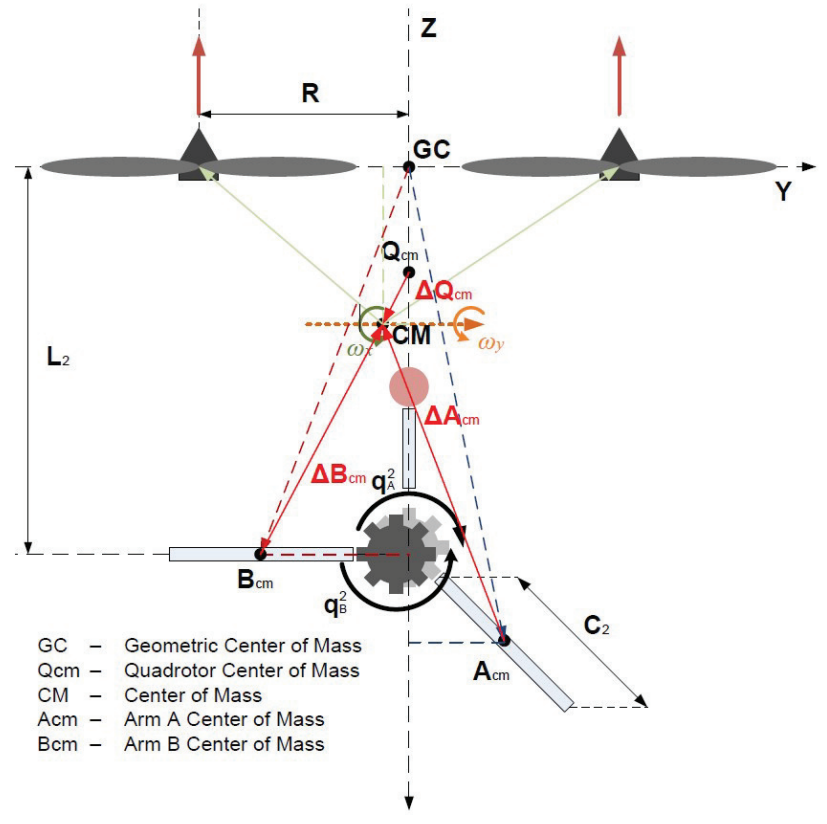

(a) Joint 1

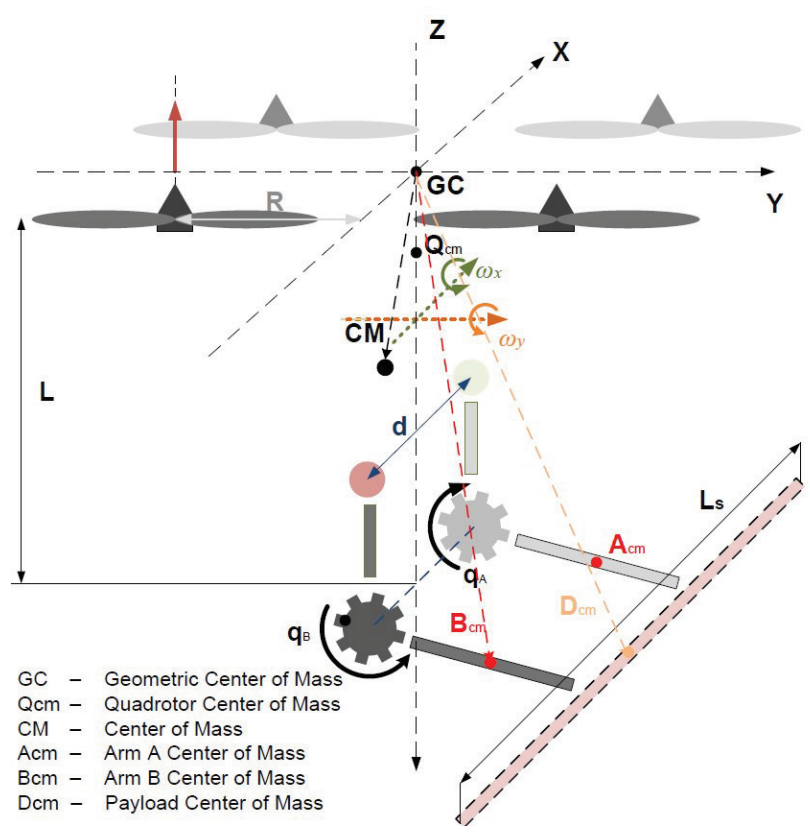

(b) Joint 2

Fig. 3: Simplified Arm Model

2, due to the fact that they carry longer part of the MMUAV arms; Furthermore, Joints 1 cause opposite effects on $I_{x x}$ and $I_{y y}$. With the arms in a horizontal position $\left(q_{A}^{1}=\right.$ $\left.q_{B}^{1}=-90^{\circ}\right), I_{y y}$ increases, while $I_{x x}$ decreases; Because the joints are perpendicular to each other, their effects are reversed; Joints 2 have a slightly larger operating range (i.e. $180^{\circ}$ instead of $120^{\circ}$ ); Finally, we conclude that Joints 2 have a similar effect on both moments of inertia, increasing them when the arms are fully extended downwards. The biggest effect is observed on the $y$ axis when Joints 1 move, and the biggest variations in moment of inertia are observed on the $x$ axis, when Joints 1 move.

\section{B. Angle Control Stability}

Control of the MM-UAV body is achieved with a standard PI-D control loop shown in Fig. 6. This type of PID implementation eliminates the potential damages to the actuators, normally experienced when driving the control difference directly through the derivation channel [25]. The major factor that affects the stability of the aircraft is the variable moment of inertia $J=I_{x x}, I_{y y}$ discussed in Sec. IV-A.

The PI-D angle control with the additional adaptive control loop is shown in Fig. 6. Its transfer function yields a 4th order characteristic polynomial $a_{4} s^{4}+a_{3} s^{3}+a_{2} s^{2}+a_{1} s+a_{0}$, where the 4th order dynamic system includes both the dynamics of the aircraft and the motor dynamics (7).

$$
G_{\alpha_{C L}}=\frac{\frac{K_{D} K_{m}}{K_{i} T_{m} J}\left(\frac{K_{p}}{K_{i}} s+1\right)}{s^{4}+\frac{1}{T_{m}} s^{3}+\frac{K_{D} K_{m}}{T_{m} J} s^{2}+\frac{K_{D} K_{m} K_{p}}{T_{m} J} s+\frac{K_{D} K_{m} K_{i}}{T_{m} J}}
$$

Coefficients $K_{D}, K_{p}$ and $K_{i}$ are PI-D respective gains and $K_{m}$ and $T_{m}$ represent propulsion system gain and motor time constant. After applying the Routh-Hurwitz stability criteria, the analytical solutions for stability conditions, which require that all coefficients be positive and that inequalities in (8) hold, are derived. Stability criteria (8) shows that, due to the dynamics introduced from the motors (i.e. $T_{m}$ ), the proportional control $K_{p}$ can drive the system unstable. In fact, only the derivative control $K_{D}$ has the sole purpose of stabilizing the system. Therefore, the Model Reference Adaptive Control loop can be applied to $K_{D}$; increasing it or decreasing depending on the overall moment of inertia.

$$
\begin{gathered}
\frac{K_{D} K_{m} K_{p}}{K_{i}}\left(1-T_{m} K_{p}\right)>J \\
K_{D} K_{m}\left(1-T_{m} K_{p}\right)>0
\end{gathered}
$$

\section{Lyapunov based MRAC control}

Judging from Fig. 6 and transfer function (7), there are four parameters in the attitude control loop of a quadrotor that change during flight and manipulation:

- $K_{m}$ - Propulsion system gain changes drastically through time, especially in load manipulation missions.

- $T_{m}$ - Propulsion system dynamics changes due to various effects as the mission progresses through time.

- $\beta$ - The aerodynamic conditions constantly change during the flight.

- $J$ - As previously discussed (IV-A) the moment of inertia changes depending on the load and arm pose.

Aerial manipulation missions mostly require steady flight conditions, for which the changes in the aerodynamic conditions as well as the aerodynamic coefficient $\beta$ can be neglected. On the other hand, if the battery power supply is kept constant throughout the mission, the variations in 


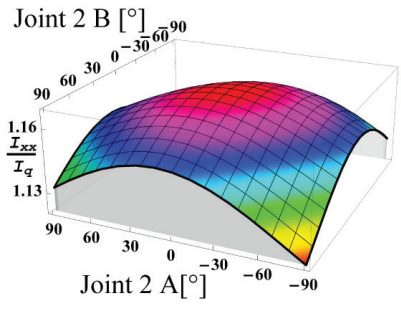

(a) $I_{y y}$ Joints $1 \mathrm{~A}$ and $1 \mathrm{~B}$

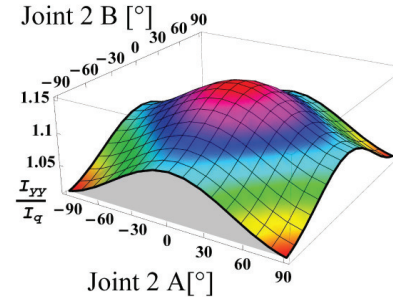

(b) $I_{x x}$ Joints $1 \mathrm{~A}$ and $1 \mathrm{~B}$

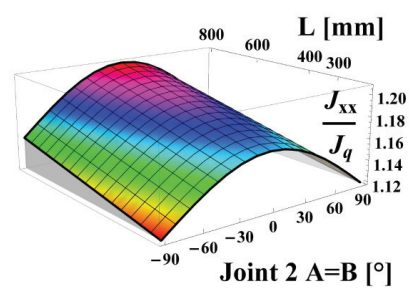

(c) $I_{y y}$ Joints $2 \mathrm{~A}$ and $2 \mathrm{~B}$

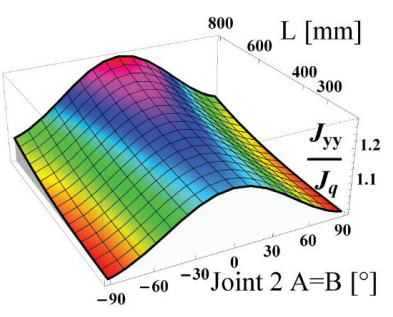

(d) $I_{x x}$ Joints $2 \mathrm{~A}$ and $2 \mathrm{~B}$

Fig. 4: Moments of inertia variations with respect to joint angle changes.

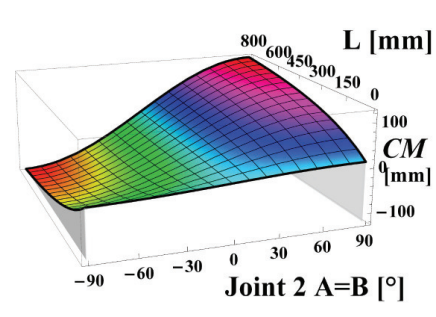

(a) $I_{y y}$ Joints $1 \mathrm{~A}$ and $1 \mathrm{~B}$

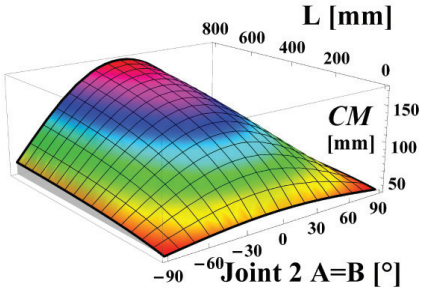

(b) $I_{x x}$ Joints $1 \mathrm{~A}$ and $1 \mathrm{~B}$
Fig. 5: Center of mass variations with respect to joint angle changes.

the $T_{m}$ can be minimized. The two remaining parameters, $J$ and $K_{m}$ diverge the most during aerial manipulation. The variations in the moment of inertia have been previously discussed. The propulsion system gain changes are mostly caused from the variations in the load mass, which changes the piecewise linearization of the quadratic relationship between the propeller thrust and the applied voltage. Apart from that, the variations in temperature and the battery depletion also change the linearized motor gain throughout the mission.

In order to adapt to the changing parameters, a Model Reference Adaptive Control mechanism is applied. Separating the highly inconsistent parameters $K_{m}$ and $J$ on one side, and fairly constant $T_{m}$ and $\beta$ on the other, allows us to write the open loop rotation speed transfer function as a class of IPT1, $y=k G(s)$ :

$$
\dot{\Theta}(s)=\left\{\frac{K_{m}}{J}\right\}\left\{\frac{1}{1+T_{m} s} \frac{1}{s}\right\} u(s)
$$

The PI-D controller is then modified so that its output is multiplied by the adaptive gain $\zeta$ and the adjustment rule for it is chosen according to:

$$
\frac{d \zeta}{d t}=-\gamma \mathbf{u}_{\mathrm{PI}-\mathrm{D}}^{T} \mathbf{e}
$$

where $\mathbf{e}$ is the error difference between the model and the actual orientation dynamics

$$
\dot{\mathbf{y}}=\left[\begin{array}{cc}
0 & 1 \\
-1 & -\frac{1}{T_{m}}
\end{array}\right] \mathbf{y}+\frac{K_{m}}{J T_{m}} \mathbf{u}
$$

Using the Lyapunov stability theory, it can be shown that such a system is uniformly asymptotically stable. Candidate
Lyapunov function is chosen:

$$
\mathbf{V}(\mathbf{e}, \zeta)=\frac{\gamma}{2} \mathbf{e}^{T} \mathbf{e}+\frac{k}{2}\left(\zeta-\zeta_{0}\right)^{2}
$$

with $\zeta_{0}$ as a steady state value of $\zeta$ and arbitrary chosen gains $\gamma$ and $k$ so that its derivative can be:

$$
\frac{d \mathbf{V}(\mathbf{e}, \zeta)}{d t}=\gamma \mathbf{e}^{T} \mathbf{e}+k\left(\zeta-\zeta_{0}\right)\left(\frac{d \zeta}{d t}+\gamma \mathbf{u} \mathbf{e}^{T} \frac{K_{m}}{T_{m} J}\right)
$$

For the chosen adaptation rule (10), it can be shown that the Lyapunov Candidate (12) is negative semi-definite if and only if $T_{m}>0$, which is always true, and $\gamma>0$ which can be arbitrary chosen. Although, the Lyapunov stability analysis sets no upper bound for the correction factor $\gamma$, it is still necessary to choose its appropriate value and to that end, the approach in [6] is chosen. A practical implementation requires that the upper and the lower bound for the adaptation gain $\zeta, \zeta_{\max }$ and $\zeta_{\min }$ are set. According to criteria (8), the range of $K_{D}$ for which the system is stable can be determined once the range of changes of the moment of inertia $J$ is known. In our case $K_{D \max }=2 K_{D 0}$ and $K_{D \min }=K_{D 0} / 2$, with $K_{D 0}$ as the nominal value of the control parameter $K_{D}$. Since the adaptation mechanism influences the system through multiplication $K_{D} \zeta$ (Fig. 6), determination of $\zeta$ maximum and minimum is straightforward, i.e. in our case $\zeta_{\max }=2$ and $\zeta_{\min }=1 / 2$. Now, one is able to estimate the range of the correction factor $\gamma$. Rewriting equation (10) gives:

$$
\zeta(t)=-\gamma \int u_{P I-D}(t) e(t) d t
$$

During the adaptation phase (Fig. 7), a set of pulses is generated by PI-D controller in order to perturb the system so that new value of the adaptation parameter can be determined, thus,

$$
\zeta(t)=-\gamma \int \delta(t)\left[y(t)-y_{m}(t)\right] d t
$$

As we already wrote, $J$ and $K_{m}$ are two parameters that are mostly influenced by aerial manipulation. Hence, including inverse Laplace transform of the system and the model (neglecting influence of $T_{m}$ ) in (15), one gets

$$
\zeta(t)=-\gamma\left(\frac{K_{m}}{J}-\frac{K_{M}}{J_{M}}\right) t+\zeta_{0}
$$

Since dynamics of the adaptation loop must be slower (usually 5 to 10 times) than the system dynamics, in case of 


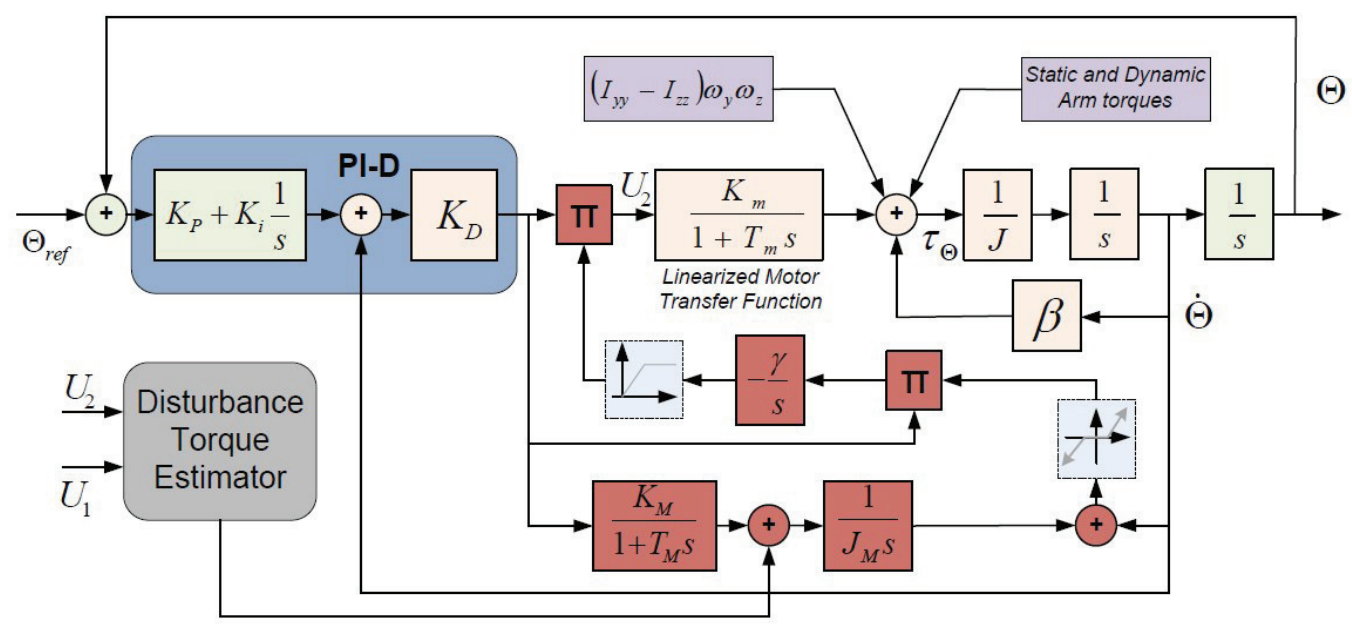

Fig. 6: Attitude Model Reference Adaptive PI-D Control

large change of parameters, the adaptation parameter should attain maximum/minimum value at $t \approx 5 \cdot\left(5 T_{m}\right)$, which gives

$$
\begin{aligned}
\zeta_{\text {max }} & \approx-\gamma\left(\frac{K_{m}}{J_{\max }}-\frac{K_{M}}{J M}\right) \cdot 25 T_{m}+\zeta_{0} \\
\zeta_{\text {min }} & \approx-\gamma\left(\frac{K_{m}}{J_{\min }}-\frac{K_{M}}{J M}\right) \cdot 25 T_{m}+\zeta_{0}
\end{aligned}
$$

Finally, lower of two values of the correction factor $\gamma$, calculated from (17) and (18), should be included in the MRAC.

Because Model Reference adaptation is highly susceptible to disturbances, one has to take into account the static and dynamic torque disturbances produced from the arm movement. That is why the disturbance torque estimator is introduced to the MRAC control schematics. Dynamic disturbances are cancelled out by using a low pass filter for the adaptation rule. Static torques however, cannot be bypassed with a filter. Static torque is caused from the shift in the center of mass of the aircraft and the gravity that affects its unbalanced body. Learning from the results in [17], one can find the unknown center of mass offset, in a least square minimization sense, simply as an average over collected data:

$$
\hat{\mathrm{CM}} \hat{\mathrm{M}}_{x}=\frac{\bar{u}_{3}}{\bar{u}_{1}}
$$

Where $\bar{u}_{1}$ and $\bar{u}_{3}$ are simply controller outputs for pitch and height control respectively. The estimation results are then fed to the MRAC model, thus minimizing the controller vulnerabilities to disturbances. Static toreque estimation works well for steady state estimation, but fails to accurately estimate the dynamic changes in the gravity torqe. Therefore, we propose adding a dead zone to the adaptation rule, in order to cancel out the estimation errors.

\section{Hybrid automaton}

To better utilize the MRAC adaptive capabilities, a hybrid system based on a switching automaton shown in IV-D is proposed. The hybrid system defines four phases of aerial manipulation missions: Flight phase, Arm deployment phase, Manipulation phase and Adaptation phase. The system starts in the flight phase, where the MM-UAV flies to the designated point. Once it arrives to the set point (i.e. $\left\|\vec{x}-x_{r e f}\right\| \leq \Delta_{x}$ ), and before switching to the manipulation stage, the aircraft repositions the arms for the manipulation. This is called the arm deployment phase. After the arms are deployed, the UAV switches to the adaptation phase and starts the self induced oscillations in order to fine tune the controller. Once ready, the MM-UAV starts the manipulation phase of the mission. The aircraft can leave the adaptation phase once $\left\|\frac{\delta \zeta}{\delta t}\right\| \leq \Delta_{\zeta}$.

During the manipulation phase, the shift in the center of mass or the change of moment of inertia could drive the vehicle unstable. Therefore, the vehicle is allowed to switch back to Adaptation phase if such a problem occurs. After successfully picking up an object and before flying back to the base, the air robot once again changes to the Adaptation phase. Because the load changed the center of mass and moment of inertia of the body, it is necessary to fine tune the controller once again, before flying off. Once tuning is complete, the aircraft switches back to flight phase and flies back to the base.

\section{Simulation}

The simulation model for MM-UAV must incorporate quadrotor dynamics and propeller aerodynamics from Sec. III, together with a complete dynamic model of the arms (Sec. II), controlling the system with a PI-D controller from Sec. IV. Figure 8 shows the layout for the simulation model used in this paper. The attitude controller takes angle reference values and quadrotor feedback signals as input. A PI-D algorithm then calculates the necessary rotor speeds that power the propeller dynamics block that produces respective torques and forces applied to the body. A recursive NewtonEuler dynamic model is used to model the arms as a disturbance to the quadrotor control loop. This recursive model calculates the torques and forces based on movements 


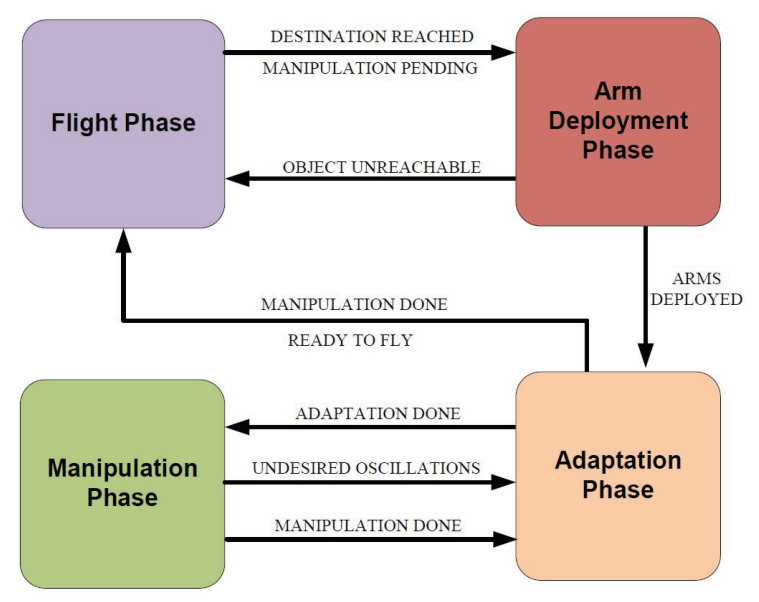

Fig. 7: Hybrid system automaton

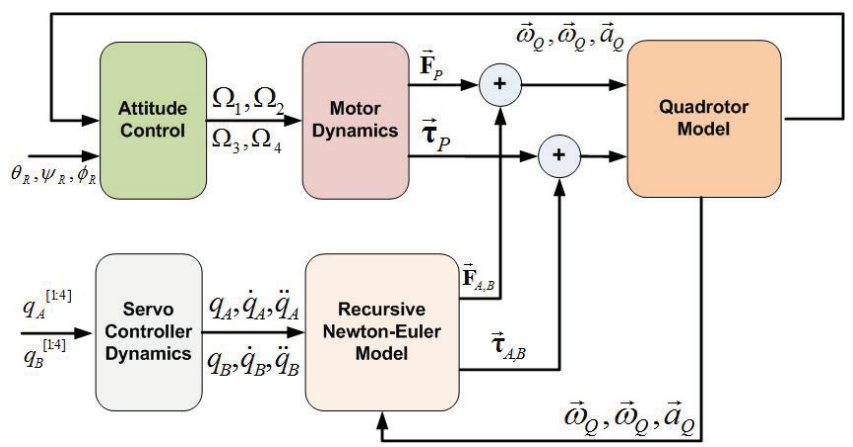

Fig. 8: Simulation Scheme
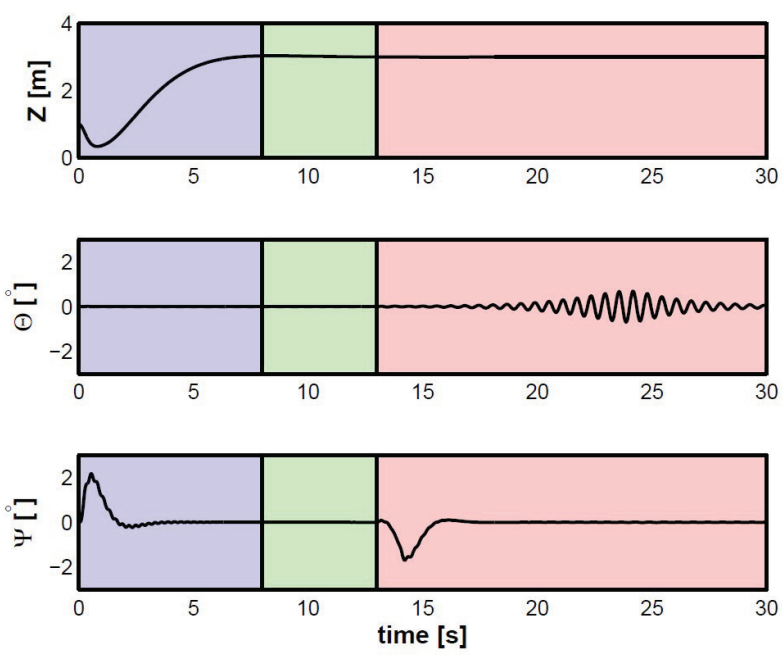

Fig. 9: Matlab Simulation (Take off with arms stowed, Oscillations settled; Deploying arms move): Roll and pitch angles

of the arms and quadrotor dynamics. Matlab was used for simulations and a recursive Newton-Euler dynamics model of the manipulators was implemented using the Robotics Toolbox [26].

In our previous work, we have showed how with a poorly

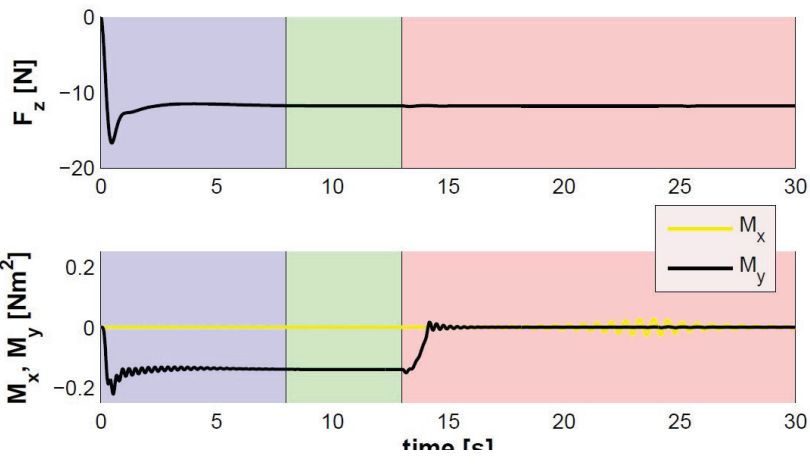

Fig. 10: Matlab Simulation (Take off with arms stowed, Oscillations settled; Deploying arms move): Propulsion system thrust and torque values

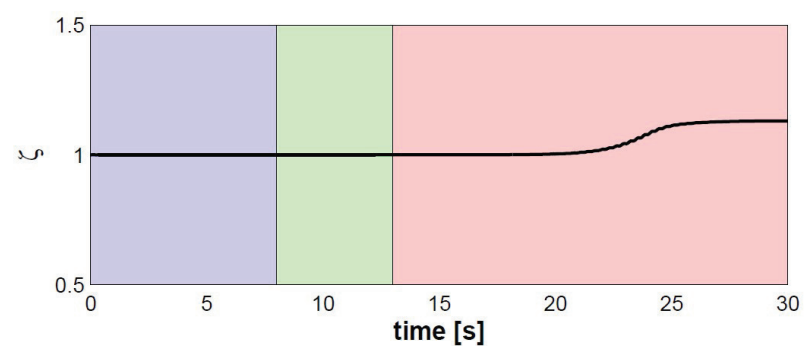

Fig. 11: The adaptive gain $\zeta$ changes as the oscillations occur, and brings the system back in the stability region
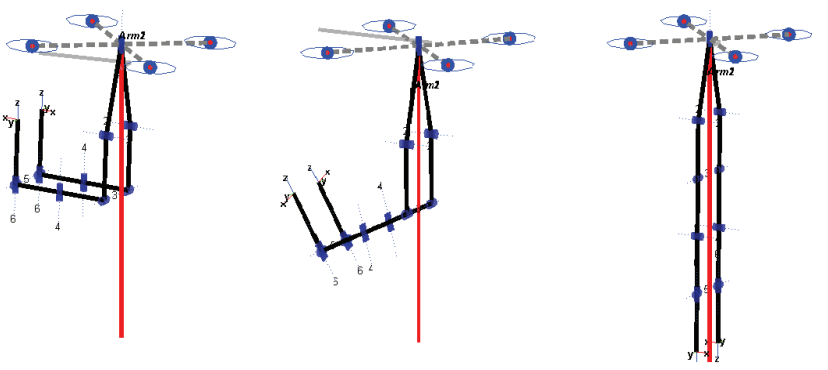

Fig. 12: Left to right, MM-UAV arm transition from stowed to fully deployed

designed PID controller air robot becomes unstable during manipulation tasks, even though it is perfectly stable during the flight [23], [24]. In this paragraph, we put the adaptive control to the test, trying to stabilize the same system from our previous work. Figures 9 and 10 show the results of one of the performed tests where the quadrotors roll controller was tuned close to the stability boundary. The aircraft takes off with arms tucked and stowed. After the vehicles settles to a hover, the arms are deployed down and fully extended (Fig. 12), thus increasing the moments of inertia. This change in the moment of inertia tries to destabilize the system and thus produces undesired oscillations in the roll angle control loop. The oscillations trigger the MRAC that changes the overall control loop gain, and therefore stabilizes the system. According to the stability criteria (8), the adaptive gain $\zeta$ needs to increase the derivative gain $K_{D}$ to account for 
the rise in $J$. Figure 11 shows how the adaptive gain $\zeta$ changes throughout the simulation,and Fig. 10 show the system response and the produced forces and torques.

\section{CONCLUSIONS}

The Model Reference Adaptive Controller proposed in this paper is used to control a multi-arm manipulating aerial vehicle implementend on a small, off-the-shelf quadrotor. Previous experimental results prooved that such a vehicle is not necessarily stable for all the possible chnages in the moment of inertia. Therefore, the additional adaptive loop for attitude stabilization is proposed. Using the Lyapunov stability theory, the controller is proven to be stable. The simulation results proved that the MRAC is capable of stabilizing the oscillations produced from the unstable PI$\mathrm{D}$ attitude control loop.

Finally a high level control system is proposed in order to ensure the saftey of the aerial manipulation missions. It is based on a switching automaton with four distinct mission phases.

In the future, the MRAC controller will be implemented and tested on the experimental platform. Also, other adaptive and robust control techniques should be tested both in simulation and experiment. Using adaptive and robust control greater flight stability should be achivede, which would enable fully dexterous manipulation.

\section{REFERENCES}

[1] C. Nicol, C. Macnab, and A. Ramirez-Serrano, "Robust adaptive control of a quadrotor helicopter," Mechatronics, vol. 21, no. 6, pp. 927 - 938, 2011.

[2] I. Palunko and R. Fierro, "Adaptive feedback controller design and quadrotor modeling with dynamic changes of center of gravity," vol. 18, no. 1, August-September 2011, pp. 2626-2631.

[3] K. Åström and B. Wittenmark, Adaptive Control, ser. Addison-Wesley Series in Electrical Engineering. Addison-Wesley, 1995. [Online]. Available: http://books.google.hr/books?id=FJ4eAQAAIAAJ

[4] I. Landau, Adaptive Control, ser. Communications and Control Engineering. Springer London, 2011. [Online]. Available: http://books.google.hr/books?id=fb1GVyJHeBgC

[5] H. Butler, Model reference adaptive control: from theory to practice, ser. Prentice Hall international series in systems and control engineering. Prentice Hall, 1992. [Online]. Available: http://books.google.hr/books?id=SUISAAAAMAAJ

[6] Z. Kovacic, S. Bogdan, and M. Puncec, "Adaptive control based on sensitivity model-based adaptation of lead-lag compensator parameters," in Industrial Technology, 2003 IEEE International Conference on, vol. 1, dec. 2003, pp. 321 - 326 Vol.1.

[7] S. Bellens, J. De Schutter, and H. Bruyninckx, "A hybrid pose / wrench control framework for quadrotor helicopters," in Robotics and Automation (ICRA), 2012 IEEE International Conference on, may 2012, pp. $2269-2274$.

[8] M. Lorenzo and N. Roberto, "Control of aerial robots: Hybrid force and position feedback for a ducted fan," Control Systems, vol. 32, no. 4, pp. 43-65, 2012.

[9] M. Lorenzo, N. Roberto, and L. Gentili, "Modelling and control of a flying robot interacting with the environment," Automatica, vol. 47, no. 12, pp. 2571-2583, 2011.

[10] M. Bernard, K. Kondak, I. Maza, and A. Ollero, "Autonomous transportation and deployment with aerial robots for search and rescue missions," J. Field Robotics, vol. 28, no. 6, pp. 914-931, 2011.

[11] M. Bisgaard, A. la Cour-Harbo, and J. Bendtsen, "Adaptive control system for autonomous helicopter slung load operations," Control Engineering Practice, vol. 18, no. 7, pp. 800-811, 2010.
[12] I. Palunko, R. Fierro, and P. Cruz, "Trajectory generation for swingfree maneuvers of a quadrotor with suspended payload: A dynamic programming approach," in Robotics and Automation (ICRA), 2012 IEEE International Conference on, may 2012, pp. 2691 -2697.

[13] L. Sandino, M. Bejar, K. Kondak, and A. Ollero, "On the use of tethered configurations for augmenting hovering stability in small-size autonomous helicopters," Journal of Intelligent and Robotic Systems, pp. 1-17, 2012. [Online]. Available: http://dx.doi.org/10.1007/s10846012-9741-2

[14] P. Pounds, D. Bersak, and A. Dollar, "The yale aerial manipulator: Grasping in flight," in Robotics and Automation (ICRA), 2011 IEEE International Conference on, may 2011, pp. 2974 -2975.

[15] P. E. I. Pounds, D. R. Bersak, and A. M. Dollar, "Grasping from the air: Hovering capture and load stability," in Proc. IEEE Int Robotics and Automation (ICRA) Conf, 2011, pp. 2491-2498.

[16] P. Pounds, D. Bersak, and A. Dollar, "Stability of small-scale uav helicopters and quadrotors with added payload mass under pid control," Autonomous Robots, vol. 33, pp. 129-142, 2012.

[17] D. Mellinger, Q. Lindsey, M. Shomin, and V. Kumar, "Design, modeling, estimation and control for aerial grasping and manipulation," in Proc. IEEE/RSJ Int Intelligent Robots and Systems (IROS) Conf, 2011, pp. 2668-2673.

[18] D. Mellinger, M. Shomin, N. Michael, and V. Kumar, "Cooperative grasping and transport using multiple quadrotors," in Proceedings of the International Symposium on Distributed Autonomous Robotic Systems, Nov 2010.

[19] Q. J. Lindsey, D. Mellinger, and V. Kumar, "Construction of cubic structures with quadrotor teams," Robotics: Science and Systems, June 2011.

[20] M. Orsag, C. Korpela, and P. Oh, "Modeling and control of MM-UAV: Mobile manipulating unmanned aerial vehicle," in Proc.International Conference on Unmanned Aircraft Systems, ICUAS, 2012.

[21] S. McMillan, D. E. Orin, and R. B. McGhee, "Efficient dynamic simulation of an underwater vehicle with a robotic manipulator," IEEE Transactions On Systems, Man, and Cybernetics, vol. 25, pp. 11941206, 1995.

[22] H. Hahn, Rigid Body Dynamics of Mechanisms: Theoretical basis, ser. Rigid Body Dynamics of Mechanisms. Springer, 2002.

[23] M. Orsag, C. Korpela, M. Pekala, and P. Oh, "Stability in aerial manipulation," in Proc. of IEEE American Control Conference (ACC), 2013, To appear.

[24] C. Korpela, M. Orsag, M. Pekala, and P. Oh, "Dynamic stability of a mobile manipulating unmanned aerial vehicle," in Proc. IEEE Int Robotics and Automation (ICRA) Conf, 2013, To Appear.

[25] N. Miskovic, Z. Vukic, M. Bibuli, M. Caccia, and G. Bruzzone, "Marine vehicles' line following controller tuning through self-oscillation experiments," in Proceedings of the 2009 17th Mediterranean Conference on Control and Automation, ser. MED '09. Washington, DC, USA: IEEE Computer Society, 2009, pp. 916-921.

[26] P. I. Corke, Robotics, Vision \& Control: Fundamental Algorithms in Matlab. Springer, 2011. 